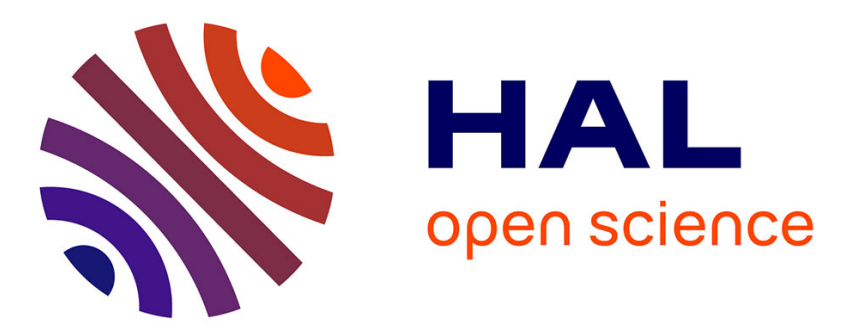

\title{
On stability of a combined Gleevec and immune model in chronic leukemia Exploiting delay system structure
}

Silviu-Iulian Niculescu, P.S. Kim, P.P. Lee, D. Levy

\section{To cite this version:}

Silviu-Iulian Niculescu, P.S. Kim, P.P. Lee, D. Levy. On stability of a combined Gleevec and immune model in chronic leukemia Exploiting delay system structure. 7th IFAC Symposium on Nonlinear Control Systems, NOLCOS 2007, Aug 2007, Pretoria, South Africa. pp.563-568. hal-02272362

\section{HAL Id: hal-02272362 \\ https://hal.science/hal-02272362}

Submitted on 27 Aug 2019

HAL is a multi-disciplinary open access archive for the deposit and dissemination of scientific research documents, whether they are published or not. The documents may come from teaching and research institutions in France or abroad, or from public or private research centers.
L'archive ouverte pluridisciplinaire $\mathbf{H A L}$, est destinée au dépôt et à la diffusion de documents scientifiques de niveau recherche, publiés ou non, émanant des établissements d'enseignement et de recherche français ou étrangers, des laboratoires publics ou privés. 


\title{
ON STABILITY OF A COMBINED GLEEVEC AND \\ IMMUNE MODEL IN CHRONIC LEUKEMIA: \\ EXPLOITING DELAY SYSTEM STRUCTURE
}

\author{
Silviu-Iulian Niculescu ${ }^{1}$ Peter S. Kim ${ }^{2}$ Peter P. Lee ${ }^{3}$ \\ Doron Levy ${ }^{4}$
}

\begin{abstract}
This paper focuses on the stability analysis of a delay-differential system encountered in modeling immune dynamics during Gleevec treatment for chronic myelogenous leukemia. A simple algorithm is proposed for the analysis of delay effects on the stability. The analysis shows that the model yields two fixed points, one stable and one unstable. The stable fixed point corresponds to some equilibrium solution in which the leukemia population is kept below the cytogenetic remission level. This result implies that, during Gleevec treatment, the resulting anti-leukemia immune response can serve to control the leukemia population. However, the rate of approach to the stable fixed point is very slow, indicating that the immune response is largely ineffective at driving the leukemia population towards the stable fixed point. Finally, a few remarks are made about possible treatment strategies that can be used to accelerate the approach of the leukemia and immune populations towards this stable equilibrium. Copyright (c)2007 IFAC.
\end{abstract}

Keywords: Delay, stability, immune dynamics, Gleevec treatment.

\section{INTRODUCTION, AND PROBLEM FORMULATION}

The stability analysis of delay differential equations (DDEs) that model biological phenomena is of recurring interest Kuang [1993], Muray [1993]. In particular, we are concerned with delay models involving the immune response to chronic myelogenous leukemia (CML). A simple DDE model for the immune re-

\footnotetext{
${ }^{1}$ S.-I. Niculescu is with Laboratoire des Signaux et Systèmes (L2S, UMR CNRS 8506), CNRS-Supélec, 3, rue Joliot Curie, 91192, Gif-sur-Yvette, France. E-mail: Silviu.Niculescullss.supelec.fr; The work of S.-I. NICULESCU was partially funded by the CNRS-US Grant: "Delays in interconnected dynamical systems: Analysis, and applications" (2005-2007)

2 P.S. Kim with the Department of Mathematics, Stanford University, California, USA. E-mail: pkimemath. stanford.edu.

3 P.P. Lee is with the Division of Hematology in the School of Medicine, Stanford University, California, USA. E-mail: pplestanford.edu

${ }_{4}$ D. Levy is with the Department of Mathematics, Stanford University, California, USA. E-mail: dlevy@math.stanford.edu. The research of D. LEVY is partially supported by the NSF under Career Grant DMS-0133511.
}

sponse in CML is due to Neiman [2002]. This work tried to explain the transition of leukemia from the stable chronic phase to the erratic accelerated and acute phases. A more recent work is of Moore and $\mathrm{Li}$ [2004]. They devised a CML model composed of a system of ODEs (without delays). The main goal was to examine which parameters are the most important in influence the success of cancer remission. Chen et al [2007] formulate a system of DDEs to model the dynamics of anti-leukemia $\mathrm{T}$ cells during Gleevec treatment for CML. The model is derived by taking the original Gleevec model of Michor et al [2005] and adding a DDE to simulate the population dynamics of anti-leukemia $\mathrm{T}$ cells. In the paper, we analyze the DDE system from the Chen model.

Gleevec works by inhibiting the abltyrosine kinase that drives CML Angestreich et al [2004] and has become the first-line therapy for CML. With Gleevec, nearly all patients achieve hematologic remission Cortes et al [2005], Lee [2000]; however, patients relapse upon Gleevec discontinuation Cortes et al [2005]. Thus, strategies to enhance Gleevec's efficacy are needed. Combining Gleevec with immunotherapy 
represents a promising strategy; however, the role of the immune response remains unclear. In vitro, imatinib renders leukemic cells immunogenic Mohty et al [2004], Sato [2003]. More specifically, low frequency (generally $<1 \%$ ) CD8+ T cell responses to 4 leukemia-associated antigens (LAAs) were detected in CML patients after therapy with Gleevec Gannage et al [2005]. Chen et al [2007] conduct further experiments to follow the time evolution of anti-leukemia $\mathrm{T}$ cells during Gleevec treatment. With this data, they formulate a DDE model based on the observed dynamics. The time delay in Chen et al [2007] corresponds to the duration of $\mathrm{T}$ cell proliferation cycles. Their mathematical model is formulated as a nonlinear delay-differential system. Its linearization around the equilibrium point of interest writes as follows:

$$
\dot{x}(t)=A_{0} x(t)+b c^{T} x(t-n \tau),
$$

under appropriate initial conditions defined on the interval $[-n \tau, 0]$, where $\tau$ represents the delay, and $n$ some averaging factor. In our case study $\left(A_{0} \in \mathbb{R}^{5 \times 5}\right.$, $b, c \in \mathbb{R}^{5 \times 1}$ ), the system (1) has the following "limit" properties: (a) the system free of delays is asymptotically stable, that is $\sigma\left(A_{0}+b c^{T}\right) \in \mathbb{C}_{-}$, and (b) the reduced system including only the "instantaneous" part is hyperbolic, that is the matrix $A_{0}$ has no eigenvalues on the imaginary axis, i.e. $\sigma(A) \cap j \mathbb{R}=\varnothing$.

The problem considered in the sequel can be resumed as follows: under the assumptions above, find simple conditions for characterizing the delay effects on stability of the linearized Gleevec model.

\section{PRELIMINARY RESULTS}

Consider now the following meromorphic function $q: \mathbb{C} \times \mathbb{R}_{+} \mapsto \mathbb{C}:$

$$
q(\lambda, h)=1-a(\lambda) e^{-\lambda h},
$$

associated to the system (1), where $a$ denotes the transfer $a(\lambda)=c^{T}\left(\lambda I_{n}-A_{0}\right)^{-1} b$, and $h=n \tau$. By exploiting the particular structure of the delayed matrix $b c^{T}$ of rank one, we have the following results (see, the full version of the paper for the complete proofs Niculescu et al [2007]):

Proposition 1. Consider the system (1). Assume that $A_{0}$ has no eigenvalues on the imaginary axis, and that $1-a(0) \neq 0$, where $a(\lambda)=c^{T}\left(\lambda I_{n}-A\right)^{-1} b$. Define now the function $f: \mathbb{R}-\{0\} \mapsto \mathbb{R}, f(\omega)=$ $1-|a(j \omega)|$. Under these conditions the following statements are true:

(i) Suppose that $f(\omega)=0$ has no strictly positive roots $\omega>0$. Then if (1) is stable at $h=0$, it remains stable for all $h \geq 0$, whereas if it is unstable at $h=0$ it remains unstable for all $h \geq 0$, and there does not exist any root crossing the imaginary axis when the delay $h$ is increased in $\mathbb{R}_{+}$. (ii) Suppose that $f(\omega)=0$ has at least one positive root, and that each root is simple. As $h$ increases, stability switches may occur. There exists a positive number $h^{*}$, such that the system (1) is unstable for all $h>h^{*}$. As $h$ varies from 0 to $h^{*}$, at most a finite number of stability switches may occur. Furthermore, if for $\omega=\omega_{0}$ one pair of roots lies on the imaginary axis, the roots will cross the imaginary axis from left to right (or from right to left) if and only if:

$$
\Re\left[\frac{a\left(j \omega_{0}\right)}{j \omega_{0} c^{T} b}\right]<0 \quad(>0) .
$$

Next, the characterization of the stability with respect to the delay is reduced to: (i) the computation of the stability crossing set $\Omega$, that is the set of all $\omega_{0} \in \mathbb{R}_{+}$ for which $q(\lambda, h)=0$ has at least one characteristic root on $j \mathbb{R}\left(\lambda=j \omega_{0}\right)$. It is important to point out that $\operatorname{card}(\Omega)$ is finite; (ii) the computation of the crossing direction for all $\omega \in \Omega$. In conclusion, we have the following computational procedure:

(1) Identification of the crossing set $\Omega$ - by a frequency sweeping test: $|a(j \omega)|$ against $\omega$. A frequency $\omega_{0} \in$ $\Omega$ if and only if $\left|a\left(j \omega_{0}\right)\right|=1$. The condition $1-$ $a(0) \neq 0$ in Proposition 1 says that $0 \notin \Omega$ (i.e. no invariant root at origin).

(2) Computation of the crossing delay set $\mathcal{T}$ : For each crossing frequency, the corresponding critical delay values are computed by using: $e^{j \omega_{0} h}=a\left(j \omega_{0}\right)$, that is ${ }^{5} \mathcal{T}\left(\omega_{0}\right)=$ :

$$
\left\{h \in \mathbb{R}_{+}: h=\frac{1}{\omega_{0}}\left[-j \log \left(a\left(j \omega_{0}\right)\right)+2 \pi \ell\right]\right\} .
$$

Finally, $\mathcal{T}$ is defined by:

$$
\mathcal{T}=\bigcup_{\omega \in \Omega} \mathcal{T}(\omega) .
$$

(3) Computation of the individual crossing directions: In our case, the crossing direction characterization reduces to the analysis of the sign of $\Re\left(-j a\left(j \omega_{0}\right)\right)$ for all $\omega_{0} \in \Omega$ since $c^{T} b=2^{n} q_{T} \bar{p}_{1}>0$.

\section{MAIN RESULTS}

Mathematical model. Chen et al [2007] formulated a DDE model combining the Gleevec dynamics of Michor et al [2005] with a T cell response. For this analysis, we exclude the possibility of Gleevec-resistance mutations and analyze the following DDE system:

$$
\begin{gathered}
\dot{y}_{0}=\left[r_{y}-d_{0}\right] y_{0}-q_{\mathrm{C}} p(C, T) y_{0}, \\
\dot{y}_{1}=a_{y} y_{0}-d_{1} y_{1}-q_{\mathrm{C}} p(C, T) y_{1}, \\
\dot{y}_{2}=b_{y} y_{1}-d_{2} y_{2}-q_{\mathrm{C}} p(C, T) y_{2}, \\
\dot{y}_{3}=c_{y} y_{2}-d_{3} y_{3}-q_{\mathrm{C}} p(C, T) y_{3},
\end{gathered}
$$

$\dot{T}=s_{\mathrm{T}}-d_{\mathrm{T}} T-p(C, T) C+2^{n} p\left(C_{n \tau}, T_{n \tau}\right) q_{\mathrm{T}} C_{n \tau}$,

\footnotetext{
${ }^{5}$ Here, "Log" denotes the principal value of the logarithm. Consequently for $|z|=1, \log (z)=j \arg (z)$ with $\arg (z) \in(-\pi, \pi]$.
} 
where

$$
\begin{aligned}
& p(C, T)=p_{0} e^{-c_{n} C} k T, \quad C=\sum_{i=0}^{3} y_{i}, \\
& C_{n \tau}=C(t-n \tau), \quad T_{n \tau}=T(t-n \tau) .
\end{aligned}
$$

The variables $y_{0}, y_{1}, y_{2}$, and $y_{3}$ denote the concentrations of leukemia hematopoietic stem cells (SC), progenitors (PC), differentiated cells (DC), and terminally differentiated cells (TC) without resistance mutations to Gleevec. The rate constants $a, b$, and $c$ are given with indices corresponding to non-resistant and resistant leukemia populations. The death rates of the four cell categories are given by $d_{0}, d_{1}, d_{2}$, and $d_{3}$ respectively. The variable $C$ denotes the total concentration of all leukemia cells. The variable $T$ denotes the concentration of anti-leukemia $\mathrm{T}$ cells. The final terms in each of the equations in (4) are of the form $q_{\mathrm{C}} p_{0} e^{-c_{n} C} k T y_{i}$. We assume the law of mass action, stating that two cell populations interact at a rate proportional to the product of their concentrations. Hence, the component $k T y_{i}$ (or $k T z_{i}$ ) is the rate of interaction between $\mathrm{T}$ cells and the leukemia subpopulation $y_{i}$ where $k$ is the kinetic coefficient. The coefficient $p_{0}$ is the probability that a T cell engages the cancer cell upon interaction, and $q_{\mathrm{C}}$ is the probability that the cancer cell dies from the $\mathrm{T}$ cell response. Furthermore, leukemia cells suppress antileukemia immune responses, and while the precise mechanism is unknown, we assume that the level of down-regulation depends on the current cancer population. In particular, we model that the probability that a $\mathrm{T}$ cell engages a cancer cell decays exponentially as a function of the cancer concentration, i.e., the probability of a productive $\mathrm{T}$ cell interaction with a cancer cell is $p_{0} e^{-c_{n} C}$ where $c_{n}$ is the rate of exponential decay due to negative pressure.

In (8), $s_{\mathrm{T}}$ denotes the constant supply rate of T cells into the system from stem cells. The second term is the natural death rate of $\mathrm{T}$ cells. The third term is the rate at which $\mathrm{T}$ cells engage leukemia cells and commit to $n$ rounds of division. The final term represents the population increase due to $n$ divisions of stimulated T cells where $\tau$ is the average duration of one division, and $C_{n \tau}$ and $T_{n \tau}$ are the time delayed cancer and $\mathrm{T}$ cell concentrations respectively. The coefficient $q_{\mathrm{T}}$ is the probability that a T cell survives the encounter with an activated leukemia cell. The method of modeling $\mathrm{T}$ cell proliferation in (8) is the same as that used in DeConde et al [2005]. Once a T cell is stimulated, it exits the collection of interacting $\mathrm{T}$ cells and reenters the system $n \tau$ time units later after $n$ divisions. This approach ensures that the doubling period of T cells saturates at $n \tau$ without a MichaelisMenten expression or other saturating terms.

Chen et al [2007] kept the parameter estimates from Michor et al [2005] where applicable, and estimated the remaining parameters based on patient data. We estimated $n, d_{\mathrm{T}}, s_{\mathrm{T}}$, and $c_{n}$ for each patient separately.
The universal estimates that apply to all patients are shown in Table A.3, and the particular parameters for the three patients, labeled $\mathrm{P} 1, \mathrm{P} 4$, and $\mathrm{P} 12$, are shown in Table A.4 in the Appendix.

Stability of fixed points. To solve for fixed points

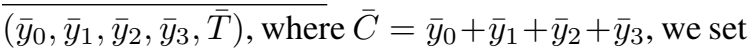
all the derivatives in (4) and (8) to zero. After a series of algebraic manipulations, we obtain the expression

$$
e^{-\kappa_{1} \bar{y}_{0}}\left(\kappa_{2}+\kappa_{3} \bar{y}_{0}\right)-\kappa_{4}=0
$$

where $\kappa_{1}=c_{n} \gamma, \kappa_{2}=s_{\mathrm{T}} / d_{\mathrm{T}}, \kappa_{3}=\left(2^{n}-1\right)\left(r_{y}-\right.$ $\left.d_{0}\right) /\left(d_{\mathrm{T}} q_{\mathrm{C}}\right), \kappa_{4}=\left(r_{y}-d_{0}\right) /\left(q_{\mathrm{C}} p_{0} k\right)$, and $\gamma=$

$\frac{a_{y}}{r_{y}+d_{1}-d_{0}}\left(1+\frac{b_{y}}{r_{y}+d_{2}-d_{0}}\left(1+\frac{c_{y}}{r_{y}+d_{3}-d_{0}}\right)\right)$.

Substituting the parameter values from Tables A.3 and A.4 and solving the implicit equation (9) numerically, we obtain values for $\bar{y}_{0}$. From $\bar{y}_{0}$, we can obtain the other components, $\bar{y}_{1}, \bar{y}_{2}, \bar{y}_{3}, \bar{T}$. We find that every patient has two fixed points (see Table A.1). These fixed points correspond to equilibrium states where the $\mathrm{T}$ cell response controls the leukemia population. For every patient, fixed point 1 represents a much more highly controlled state where leukemia is kept below the cytogenetic remission level of about $0.01 \mathrm{k} / \mu \mathrm{L}$. Thus, for medical purposes, fixed point 1 is a much more desirable ending place for the dynamical system. Next, for any fixed point $\left(\bar{y}_{0}, \bar{y}_{1}, \bar{y}_{2}, \bar{y}_{3}, \bar{T}\right)$, with $\bar{C}=\bar{y}_{0}+\bar{y}_{1}+\bar{y}_{2}+\bar{y}_{3}$, the linearization of (4) to (8) can be written as $\dot{\mathbf{x}}(t)=A \mathbf{x}(t)+b c^{T} \mathbf{x}(t-n \tau)$ where $\mathbf{x}=\left(y_{0}, y_{1}, y_{2}, y_{3}, T\right)^{\mathrm{T}}$ and

$$
\begin{array}{ll}
A_{11}=r_{y}-d_{0}-q_{\mathrm{C}} \bar{p}+\bar{q}_{1} \bar{y}_{0}, & A_{21}=a_{y}+\bar{q}_{1} \bar{y}_{1}, \\
A_{12}=\bar{q}_{1} \bar{y}_{0}, & A_{22}=-d_{1}-q_{\mathrm{C}} \bar{p}+\bar{q}_{1} \bar{y}_{1}, \\
A_{13}=\bar{q}_{1} \bar{y}_{0}, & A_{23}=\bar{q}_{1} \bar{y}_{1}, \\
A_{14}=\bar{q}_{1} \bar{y}_{0}, & A_{24}=\bar{q}_{1} \bar{y}_{1}, \\
A_{15}=-\bar{q}_{2} \bar{y}_{0}, & A_{25}=-\bar{q}_{2} \bar{y}_{1}, \\
& A_{41}=\bar{q}_{1} \bar{y}_{3}, \\
A_{31}=\bar{q}_{1} \bar{y}_{2}, & A_{42}=\bar{q}_{1} \bar{y}_{3}, \\
A_{32}=b_{y}+\bar{q}_{1} \bar{y}_{2}, & A_{43}=c_{y}+\bar{q}_{1} \bar{y}_{3}, \\
A_{33}=-d_{2}-q_{\mathrm{C}} \bar{p}+\bar{q}_{1} \bar{y}_{2}, & A_{44}=-d_{3}-q_{\mathrm{C}} \bar{p}+\bar{q}_{1} \bar{y}_{3}, \\
A_{34}=\bar{q}_{1} \bar{y}_{2}, & \\
A_{35}=-\bar{q}_{2} \bar{y}_{2}, & \\
& \\
A_{51}=-\bar{p}\left(1-c_{n} \bar{C}\right), & \\
A_{52}=-\bar{p}\left(1-c_{n} \bar{C}\right), & \\
A_{53}=-\bar{p}\left(1-c_{n} \bar{C}\right), & \\
A_{54}=-\bar{p}\left(1-c_{n} \bar{C}\right), & \\
A_{55}=-d_{\mathrm{T}}-\bar{p}_{1}, & \\
b^{T}=(00001) \text { and } c^{T}=2^{n} q_{\mathrm{T}} \cdot \\
\cdot\left(\bar{p}\left(1-c_{n} \bar{C}\right) \bar{p}\left(1-c_{n} \bar{C}\right) \bar{p}\left(1-c_{n} \bar{C}\right) \bar{p}\left(1-c_{n} \bar{C}\right) \bar{p}_{1}\right) .
\end{array}
$$

Next, $p(\lambda, \tau):=p_{0}(\lambda)+p_{1}(\lambda) e^{-n \tau \lambda}$, where

$p_{0}(\lambda)=p_{00}+p_{01} \lambda+p_{02} \lambda^{2}+p_{03} \lambda^{3} p_{04} \lambda^{4}+p_{05} \lambda^{5}$, $p_{1}(\lambda)=p_{10}+p_{11} \lambda+p_{12} \lambda^{2}+p_{13} \lambda^{3} p_{14} \lambda^{4}$

and $n$ is given in Table A.4 for each patient (see Table A.5 for the corresponding coefficients). Let $f(\omega)=1-|a(j \omega)|$, where $a(j \omega)=-p_{1}(j \omega) / p_{0}(j \omega)$ as in Proposition 1. If $f(\omega)=0$ has a root $\omega$, then $|a(j \omega)|=1$. For a given $\omega_{0}$, the value of the analytic function $q\left(j \omega_{0}, n \tau\right)$, given in (2), equals zero when

$$
\tau=\frac{\arg \left(a\left(j \omega_{0}\right)\right)}{\omega_{0} n} .
$$


Take $\arg \left(a\left(j \omega_{0}\right)\right) \in[0,2 \pi)$ to find the smallest positive $\tau$. By (3) from Proposition 1, the crossing direction as $\tau$ increases is given by the sign of

$$
\Re\left[\frac{a\left(j \omega_{0}\right)}{j \omega_{0} c^{T} b}\right] .
$$

If (2) is satisfied, then $a\left(j \omega_{0}\right)=e^{j \omega_{0} n \tau}$. Furthermore, $\omega_{0}>0$ and $c^{T} b=\bar{p}_{1}>0$, so the sign of (11) is the same as the sign of $\sin \left(\omega_{0} n \tau\right)$. In our case, all crossings are from the left to the right (i.e. toward instability) as $\tau$ increases.

For all three patients, fixed point 1 gives one positive solution $\omega_{0}$, so there is one family of stability crossings given by (10). On the other hand, for fixed point 2, we always have $|a(j \omega)|<1$, so there are no stability crossings as $\tau$ increases. Also, for $\tau=0$, fixed point 1 is stable for all three patients, while fixed point 2 is unstable. This information is summarized in Table A.2. Note that, for fixed point 1, the first three eigenvalues are almost identical across the three patients. From our numerical computations the eigenvalues match up to the first five significant digits, but begin to vary after the sixth digit. Nonetheless, this phenomenon is surprising, especially since the coefficients of the quasipolynomial $p(\lambda, \tau)$ are different across the three patients. (See Table A.5. Hence, it appears that the first three eigenvalues of the characteristic polynomial, obtained when $\tau=$, are highly insensitive to perturbations in the parameters $s_{\mathrm{T}}, d_{\mathrm{T}}$, and $c_{n}$. We are not sure why this is the case, so we leave this problem for a future study. For all patients $\mathrm{P} 1, \mathrm{P} 4$, and $\mathrm{P} 12$, fixed point 1 is stable for a wide range of $\tau$ values. Biologically $\tau$ represents the duration in days of one round of $\mathrm{T}$ cell division and is estimated to be around one day Janeway et al [2005], Lee [2006], Luzyanina et al [2004]. Hence, from a biological point of view, the upper limit $\tau_{c}$ for stablity is unattainable, and hence, the stability of fixed point 1 is independent of any reasonable error in the estimation of the delay $\tau$. However, note that the real part of rightmost eigenvalues for fixed point 1 are of the order negative $10^{-3}$ to $10^{-4}$. Hence, the solution converges to the fixed point very slowly. As $\tau$ increases from 0 to $\tau_{c}$ and greater, the rightmost roots of $p$ travel from $\mathbb{C}_{-}$across $j \mathbb{R}$ and into $\mathbb{C}_{+}$. Figure A. 1 shows the trajectories of the rightmost characteristic roots for fixed points 1 of $\mathrm{P} 1, \mathrm{P} 4$, and P12.

\section{INTERPRETATIONS AND CONCLUSIONS}

We find that in each of the three cases, P1, P4, and $\mathrm{P} 12$, fixed point 1 is robustly stable for a wide range of delays $\tau$. The total leukemia concentrations for all fixed points 1 fall well below the level of cytogenetic remission. More accurately, the total leukemia concentrations fall at around $10^{-3} \mathrm{k} / \mu \mathrm{L}$, which is between cytogenetic $\left(10^{-2}\right)$ and molecular $\left(10^{-4}\right)$ remission. This robust stability implies that, as long as conditions remain similar over time, the immune response is able to control the leukemia population and gradually drive it to a sustained cytogenetic remission. On the other hand, the rate of convergence to this fixed point is very slow, with an exponential convergence rate on the order of at most $10^{-3} /$ day. From a medical perspective, this rate of convergence is inadequate. In addition, biological conditions are likely to change over the extended time frame necessary for convergence. Therefore, one method to improve the effectiveness of Gleevec therapy is to induce the dynamical system to settle down at the stable fixed point as quickly as possible. This may be done by continually measuring a patient's leukemia load and modulating the Gleevec dosage accordingly to ensure a stabilized cancer population without high amplitude oscillations. Such a method would involve formulating a delayedfeedback control mechanism, in which the effects of altering Gleevec dosages would become apparent only after a time delay. This delay would have to be accounted for when formulating a control strategy.

As a future work, we plan to develop a possible delayed-feedback control mechanism to optimize the effectiveness of Gleevec treatment on chronic myelogenous leukemia. Furthermore, we intend to extend the analysis to include the dependence of asymptotic stability on the average number $n$ of T cell divisions after stimulation. Eventually, we plan to conduct a more extensive sensitivity analysis to determine the sensitivity of the model to key parameters.

\section{REFERENCES}

Angstreich, G. R., Smith, B. D., Jones, R. J.: Treatment options for chronic myeloid leukemia: imatinib versus interferon versus allogeneic transplant. Curr. Opin. Oncol. 16 (2004) 95-99.

Antia, R., Bergstrom, C.T., Pilyugin, S.S., Kaech, S.M., Ahmed, R.: Models of CD8+ responses: 1. What is the antigen-independent proliferation program. J Theor Biol. 221 (2003) 585-598.

Chen, C. I-U., Kim, P.S., Maecker, H.T., Levy, D., Lee, P.P.: Dynamics and Impact of the immune response to chronic myelogenous leukemia. Submitted for publication.

Cortes, J. et al.: Molecular responses in patients with chronic myelogenous leukemia in chronic phase treated with imatinib mesylate. Clin. Cancer Res.11 (2005) 3425-3432

De Boer, R.J., Homann, D., Perelson, A.S.: Different dynamics of CD4+ and CD8+ T cell responses during and after acute lymphocytic choriomeningitis virus infection. J Immunol. 171 (2003) 3928-3935.

DeConde, R., Kim, P.S., Levy, D., Lee, P.P.: Posttransplantation dynamics of the immune response to chronic myelogenous leukemia. J Theor Biol. 236 (2005) 39-59.

Gannage, M. et al.: Ex vivo characterization of multiepitopic tumor-specific CD8 T cells in patients with chronic myeloid leukemia: implications for vaccine 
development and adoptive cellular immunotherapy. J. Immunol. 174 (2005) 8210-8218.

Janeway, C.A. Jr., Travers, P., Walport, M., Shlomchik, M.J.: Immunobiology : the immune system in health and disease 6th ed. (Garland Science Publishing: New York, NY 2005.)

Kuang, Y.: Delay differential equations with applications in population dynamics (Academic Press, Boston, 1993).

Lee, P.P.: Unpublished data, 2006.

Lee, S. J.: Chronic myelogenous leukaemia. Br. J. Haematol. 111 (2000) 993-1009.

Luzyanina, T., Engelborghs, K., Ehl, S., Klenerman, P., Bocharov, G.: Low level viral persistence after infection with LCMV: a quantitative insight through numerical bifurcation analysis. Math. Biosci. 173 (2004) 1-23.

Michor, F., Hughes, T.P., Iwasa, Y., Branford, S., Shah, N.P., Sawyers, C.L., Nowak, M.A.: Dynamics of chronic myeloid leukaemia. Nature 435 (2005) 1267-1270.

Mohty, M. et al.: Imatinib and plasmacytoid dendritic cell function in patients with chronic myeloid leukemia. Blood 103 (2004) 4666-4668.

Moore, H., Li, N.K.: A mathematical model for chronic myelogenous leukemia (CML) and T cell interaction. J. Theor. Biol. 227 (2004) 513-523.

Murray, J.D.: Mathematical Biology (BioMath. 18, Springer: Berlin, 2nd Edition, 1993).

Niculescu, S.-I., Kim, P.S., Lee, P.P. and Levy, D. (2007). On stability of a combined Gleevec and immune model in chronic leukemia: Exploiting delay system structure. (full version of the paper).

Neiman, B.: A Mathematical Model of Chronic Myelogenous Leukemia. M.Sc. Dissertation (2002), University College, Oxford University, UK.

Sato, N. et al.: The effects of STI571 on antigen presentation of dendritic cells generated from patients with chronic myelogenous leukemia. Hematol. Oncol. 21 (2003) 67-75. 
Appendix A. APPENDIX: PARAMETER TABLES, COEFFICIENTS AND FIGURES

\begin{tabular}{|c||l|l|l|l|l|l|}
\hline \multicolumn{1}{|c||}{ Patient } & \multicolumn{2}{|c|}{ P1 } & \multicolumn{2}{c|}{ P4 } & \multicolumn{1}{c|}{ P12 } \\
\hline Fixed pt. & \multicolumn{1}{|c||}{1} & \multicolumn{1}{|c|}{2} & \multicolumn{1}{c|}{1} & \multicolumn{1}{c|}{1} & \multicolumn{1}{c|}{2} \\
\hline \hline $\bar{y}_{0}$ & $1.3312 \mathrm{e}-5$ & $1.5978 \mathrm{e}-1$ & $1.2844 \mathrm{e}-5$ & $1.7953 \mathrm{e}-2$ & $6.6409 \mathrm{e}-5$ & $1.5597 \mathrm{e}-1$ \\
$\bar{y}_{1}$ & $1.8127 \mathrm{e}-5$ & $2.1757 \mathrm{e}-1$ & $1.7490 \mathrm{e}-5$ & $2.4447 \mathrm{e}-2$ & $9.0429 \mathrm{e}-5$ & $2.1238 \mathrm{e}-1$ \\
$\bar{y}_{2}$ & $5.5883 \mathrm{e}-6$ & $6.7074 \mathrm{e}-2$ & $5.3918 \mathrm{e}-6$ & $7.5365 \mathrm{e}-3$ & $2.7878 \mathrm{e}-5$ & $6.5475 \mathrm{e}-2$ \\
$\bar{y}_{3}$ & $7.3943 \mathrm{e}-4$ & 8.8752 & $7.1344 \mathrm{e}-4$ & $9.9723 \mathrm{e}-1$ & $3.6888 \mathrm{e}-3$ & 8.6636 \\
$\bar{T}$ & $9.5908 \mathrm{e}-3$ & $9.1113 \mathrm{e}+1$ & $9.6332 \mathrm{e}-3$ & $1.2894 \mathrm{e}+1$ & $9.6126 \mathrm{e}-3$ & $1.2247 \mathrm{e}+1$ \\
\hline
\end{tabular}

Table A.1. Fixed points for patients P1, P4, and P12.

\begin{tabular}{|c|c|c|c|}
\hline Patient & P1 & $\mathrm{P} 4$ & P12 \\
\hline & \multicolumn{3}{|c|}{ Fixed point 1} \\
\hline$\omega_{c}$ & $6.4626 \mathrm{e}-4$ & $1.7701 \mathrm{e}-3$ & $7.9424 \mathrm{e}-4$ \\
\hline$\tau_{c}$ & 185.75 & 86.672 & 291.02 \\
\hline \multirow{5}{*}{$\begin{array}{l}\text { Eigenvalues/ } \\
\text { roots of } p(\lambda, \tau) \\
\text { for } \tau=0\end{array}$} & $-7.5575 \mathrm{e}-1$ & -7.5575 e- 1 & $-7.5575 \mathrm{e}-1$ \\
\hline & $-4.3250 \mathrm{e}-2$ & $-4.3250 \mathrm{e}-2$ & $-4.3250 \mathrm{e}-2$ \\
\hline & $-1.1750 \mathrm{e}-2$ & $-1.1750 \mathrm{e}-2$ & $-1.1750 \mathrm{e}-2$ \\
\hline & $-4.5164 \mathrm{e}-4+5.6771 \mathrm{e}-4 \mathrm{j}$ & $-6.9802 \mathrm{e}-4+1.9732 \mathrm{e}-3 \mathrm{j}$ & -6.2610 e-3 \\
\hline & $-4.5164 \mathrm{e}-4-5.6771 \mathrm{e}-4 \mathrm{j}$ & $-6.9802 \mathrm{e}-4-1.9732 \mathrm{e}-3 \mathrm{j}$ & $-3.3455 \mathrm{e}-4$ \\
\hline & \multicolumn{3}{|c|}{ Fixed point 2} \\
\hline$\omega_{c}$ & None & None & None \\
\hline$\tau_{c}$ & None & None & None \\
\hline \multirow{5}{*}{$\begin{array}{l}\text { Eigenvalues/ } \\
\text { roots of } p(\lambda, \tau) \\
\text { for } \tau=0\end{array}$} & $-7.5490 \mathrm{e}-1$ & -7.5507 e- 1 & $-7.5507 \mathrm{e}-1$ \\
\hline & $-4.2401 \mathrm{e}-2$ & $-4.2571 \mathrm{e}-2$ & $-4.2575 \mathrm{e}-2$ \\
\hline & $-1.0901 \mathrm{e}-2$ & $-1.1071 \mathrm{e}-2$ & -1.1075 e-2 \\
\hline & -9.8591 e- 4 & $-2.0606 \mathrm{e}-3$ & $-6.8221 \mathrm{e}-3$ \\
\hline & $4.6607 \mathrm{e}-2$ & $3.8423 \mathrm{e}-2$ & $3.8059 \mathrm{e}-2$ \\
\hline
\end{tabular}

Table A.2. Roots $\omega_{c}$ and delays $\tau_{c}$ correspond to the first stability crossing. In each case, for fixed point 1 , there is one family of stability crossings, and the eigenvalues for $\tau=0$ have only negative real parts. Hence, fixed point 1 is stable for $\tau \in\left[0, \tau_{c}\right)$. In each case, for fixed point 2 , there is no stability crossing and eigenvalues for $\tau=0$ has one root with positive real part. Hence, fixed point 2 is unstable for all non-negative $\tau$.

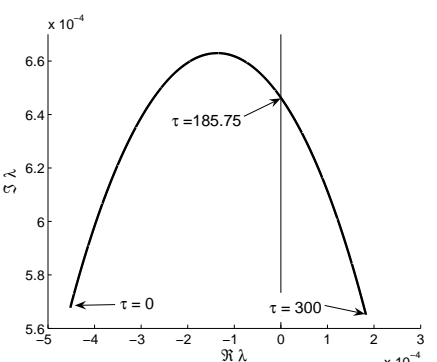

P1

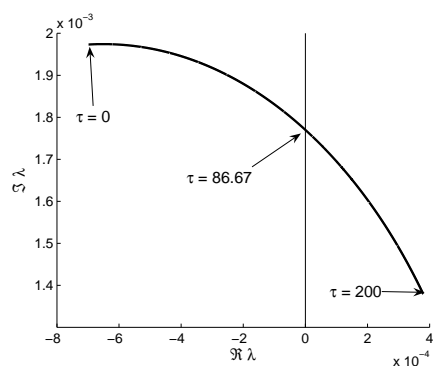

P4

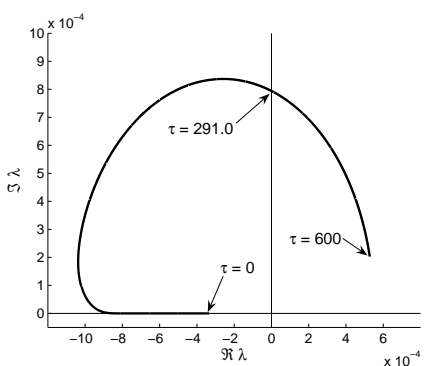

P12

Fig. A.1. Trajectories of rightmost eigenvalues of fixed points 1 as $\tau$ increases. Conjugate eigenvalues (not shown) follow analogous paths in the lower half of the complex plane. 


\begin{tabular}{|c|c|c|c|}
\hline Param & Description & Estimate & Ref./Reasoning \\
\hline $\begin{array}{l}\lambda d_{0} \\
\lambda d_{1} \\
\lambda d_{2} \\
\lambda d_{3}\end{array}$ & $\begin{array}{l}\text { SC death rate } \\
\text { PC death rate } \\
\text { DC rate } \\
\text { TC death rate }\end{array}$ & $\begin{array}{l}0.003 \lambda / \text { day } \\
0.008 \lambda \\
0.05 \lambda \\
\lambda\end{array}$ & Michor et al [2005] \\
\hline $\begin{array}{l}r_{y} \\
a_{y} \\
b_{y} \\
c_{y}\end{array}$ & $\begin{array}{l}\text { Growth rate for nonresistant cells } \\
\text { Rates without Gleevec treatment }\end{array}$ & $\begin{array}{l}0.008 / \text { day } \\
1.6 \\
10 \\
100 \\
\end{array}$ & Michor et al [2005] \\
\hline $\begin{array}{l}a_{y}^{\prime} \\
b_{y}^{\prime} \\
c_{y}^{\prime} \\
\end{array}$ & Rates during Gleevec treatment & $\begin{array}{l}a_{y} / 100 \\
b_{y} / 750 \\
c_{y} \\
\end{array}$ & Michor et al [2005] \\
\hline $\begin{array}{l}k \\
p_{0} \\
q_{\mathrm{C}} \\
q_{\mathrm{T}} \\
\tau \\
\end{array}$ & $\begin{array}{l}\text { Kinetic coefficient } \\
\text { Prob. T cell engages cancer cell } \\
\text { Prob. cancer cell dies from encounter } \\
\text { Prob. T cell survives encounter } \\
\text { Duration of one division }\end{array}$ & $\begin{array}{l}1(\mathrm{k} / \mu \mathrm{L})^{-1} \mathrm{day}^{-1} \\
0.8 \\
0.75 \\
0.5 \\
1 \text { day }\end{array}$ & $\begin{array}{l}\text { DeConde et al [2005], Luzyanina et al [2004] } \\
\text { Lee [2006] } \\
\text { Janeway et al [2005], Lee [2006], Luzyanina et al [2004] }\end{array}$ \\
\hline $\begin{array}{l}y_{0}(0) \\
y_{1}(0) \\
y_{2}(0) \\
y_{3}(0)\end{array}$ & $\begin{array}{l}\text { Initial conc. } \\
\text { Initial concentration } \\
\text { Initial concentration } \\
\text { Initial concentration } \\
\end{array}$ & $\begin{array}{l}a_{y} y_{0} / d_{1} \\
b_{y} y_{1} / d_{2} \\
c_{y} y_{2} / d_{3} \\
\end{array}$ & $\begin{array}{l}\text { Determined by patient data } \\
\text { Steady state } \\
\text { Steady state } \\
\text { Steady state }\end{array}$ \\
\hline $\begin{array}{l}z_{0}(0) \\
z_{1}(0) \\
z_{2}(0) \\
z_{3}(0)\end{array}$ & $\begin{array}{l}\text { Initial concentration } \\
\text { Initial concentration } \\
\text { Initial concentration } \\
\text { Initial concentration }\end{array}$ & $\begin{array}{l}0 \text { or } 10^{-9} \\
a_{z} z_{0} / d_{1} \\
b_{z} z_{1} / d_{2} \\
c_{z} z_{2} / d_{3} \\
\end{array}$ & $\begin{array}{l}\text { Correspond to values in Michor et al [2005] } \\
\text { Steady state } \\
\text { Steady state } \\
\text { Steady state }\end{array}$ \\
\hline$T(0)$ & Initial concentration & $s_{\mathrm{T}} / d_{\mathrm{T}}$ & Steady state \\
\hline
\end{tabular}

Table A.3. Universal parameter estimates for all patients.

\begin{tabular}{|l|l|c|c|c|}
\hline Param & Description (units) & \multicolumn{3}{|c|}{ Estimates (P1, P4, P12) } \\
\hline \hline$y_{0}(0)$ & Initial conc. of leukemia stem cells $(\mathrm{k} / \mu \mathrm{L})$ & $7.6 \times 10^{-6}$ & $2.4 \times 10^{-6}$ & $1.2 \times 10^{-5}$ \\
$n$ & Average \# of T cell divisions & 2.2 & 1.2 & 1.17 \\
$d_{\mathrm{T}}$ & T cell death rate & 0.001 & 0.0022 & 0.007 \\
$s_{\mathrm{T}}$ & T cell supply rate & $2 \times 10^{-6}$ & $9 \times 10^{-7}$ & $3.08 \times 10^{-5}$ \\
$c_{n}$ & Decay rate of immune responsitivity & 1 & 7 & 0.8 \\
\hline
\end{tabular}

Table A.4. Parameters for patients P1, P4, and P12.

\begin{tabular}{|c|c|c|c|c|c|c|}
\hline Patient & \multicolumn{2}{|c|}{ P1 } & \multicolumn{2}{|c|}{ P4 } & \multicolumn{2}{|c|}{ P12 } \\
\hline Fixed pt. & 1 & 2 & 1 & 2 & 1 & 2 \\
\hline$p_{00}$ & -1.3724 e- 9 & -1.7573 e- 8 & -1.3423 e- 9 & -3.0756 e- 8 & -6.8706 e-9 & $-1.0401 \mathrm{e}-7$ \\
\hline$p_{01}$ & $4.7040 \mathrm{e}-7$ & -1.7700 e-5 & $9.1513 \mathrm{e}-7$ & -1.6032 e-5 & $3.1151 \mathrm{e}-6$ & -2.1081 e-5 \\
\hline$p_{02}$ & $4.4917 \mathrm{e}-4$ & -1.5173 e-3 & 4.9758 e- 4 & -1.1523 e-3 & 7.9328 e- 4 & -9.2864 e- 4 \\
\hline$p_{03}$ & $4.3381 \mathrm{e}-2$ & $4.3982 \mathrm{e}-3$ & $4.4313 \mathrm{e}-2$ & $1.2502 \mathrm{e}-2$ & $5.0221 \mathrm{e}-2$ & $2.0000 \mathrm{e}-2$ \\
\hline$p_{04}$ & $8.1237 \mathrm{e}-1$ & $7.6335 \mathrm{e}-1$ & $8.1351 \mathrm{e}-1$ & $7.7361 \mathrm{e}-1$ & $8.2082 \mathrm{e}-1$ & $7.8314 \mathrm{e}-1$ \\
\hline$p_{05}$ & 1 & 1 & 1 & 1 & 1 & 1 \\
\hline$p_{10}$ & $1.5745 \mathrm{e}-9$ & $1.5403 \mathrm{e}-9$ & 3.0247 e- 9 & 2.5814 e- 9 & 7.6750 e- 9 & $1.1576 \mathrm{e}-8$ \\
\hline$p_{11}$ & $-1.0134 \mathrm{e}-7$ & -8.8209 e- 8 & $-1.9467 \mathrm{e}-7$ & $-1.5171 \mathrm{e}-7$ & -4.9396 e- 7 & -6.8072 e-7 \\
\hline$p_{12}$ & -2.6675 e-5 & -2.7680 e-5 & -5.1243 e-5 & -4.5825 e-5 & $-1.3003 \mathrm{e}-4$ & $-2.0544 \mathrm{e}-4$ \\
\hline$p_{13}$ & $-5.7395 \mathrm{e}-4$ & -6.1609 e-4 & $-1.1026 \mathrm{e}-3$ & -1.0130 e-3 & -2.7978 e-3 & $-4.5407 \mathrm{e}-3$ \\
\hline$p_{14}$ & -7.1298 e- 4 & $-7.6776 \mathrm{e}-4$ & $-1.3697 \mathrm{e}-3$ & $-1.2615 \mathrm{e}-3$ & -3.4755 e- 3 & -5.6548 e-3 \\
\hline
\end{tabular}

Table A.5. Coefficients of the quasipolynomials for patients P1, P4, and P12. 